\title{
Relationship Between Vitamin D Deficiency and
}

\section{Chronic Tension-type Headache}

\author{
Aydın Çağaç \\ Department of Neurology, Van Yuzuncu Yil University, Van, Turkey
}

\begin{abstract}
Vitamin D Deficiency is a possible factor involved in the etiology of chronic tension-type headache (CTTH). Vitamin D is directly associated with lifestyle and eating habits and affects a large proportion of the general population. The aim of this study was to investigate the role of vitamin D deficiency in CTTH.

Patients aged 18 years or older that presented to our Neurology polyclinic with CTTH between December 1, 2017 and March 31, 2018 were reviewed retrospectively. Patients were assigned into three groups based on their Vitamin D levels: (I) deficiency state: $<10$ $\mathrm{ng} / \mathrm{mL}$, (II) insufficiency state: 10-25 ng/mL, and (III) optimal state: $25-70 \mathrm{ng} / \mathrm{mL}$. The groups were compared with regard to age, gender, and Vitamin D levels.

The study included a total of 782 patients comprising $32 \%$ men and $68 \%$ women with a mean age of 34 years. Based on their serum Vitamin D levels, $482(61.6 \%)$ patients were classified into Group I ( $<10 \mathrm{ng} / \mathrm{mL}), 211(27.0 \%)$ patients into Group II (10-25 $\mathrm{ng} / \mathrm{mL})$, and $89(11.4 \%)$ patients into Group III $(>25 \mathrm{ng} / \mathrm{mL})$ and a significant difference was found among the three groups with regard to serum Vitamin D levels $(\mathrm{p}<0.01)$.

Vitamin D deficiency is an increasingly major health problem due to modern lifestyle. Accordingly, Vitamin D deficiency should be considered in patients presenting with CTTH.
\end{abstract}

Key Words: Chronic headache, vitamin deficiency, vitamin D

\section{Introduction}

Tension-type headache (TTH) is the most prevalent form of primary headache with a prevalence of 20$30 \%$ in the general population. TTH is mostly seen in women aged between 20 and 40 years. In the International Classification of Headache Disorders Third Edition Beta Version (ICHD-3 $\beta$ ), CTTH is defined as a pressing or tightening band-like headache mostly occurring in bilateral temporal and frontal regions. The pain in CTTH may appear in the form of attacks, lasting between $30 \mathrm{~min}$ and 15 days, and can vary in severity from mild to moderate. This pain does not prevent normal daily work and is not aggravated by routine physical activity. Moreover, the pain can be accompanied by photophobia or phonophobia and not by nausea or vomiting (1-5).

Vitamin D is synthesized in the skin by the effects of sunlight. This vitamin has two forms including cholecalciferol (Vitamin D3) which is synthesized in the skin and ergocalciferol (Vitamin D2) which is ingested from the diet and from supplements. Adequate synthesis of Vitamin D3 or its adequate ingestion from the diet is highly essential to lifelong muscle and bone health. Vitamin D deficiency remains a widespread health problem arising from from low intake of dietary vitamin D and from reduced sunlight exposure associated with a sedentary lifestyle, excessive use of sun block creams, and seasonal variation (6-9).

Muscle tenderness is the most crucial factor in the formation of TTH. The pathophysiology of TTH is associated with the increased tenderness in pericranial muscles. As a result of diffuse muscle pain, the tenderness in pericranial muscles may lead to neckshoulder muscle tenderness $(5,10,11)$. In a previous retrospective study, Prakash et al. reported that the patients with CTTH had a high incidence of Vitamin $\mathrm{D}$ deficiency. Moreover, some other studies showed a strong relationship between decreased serum Vitamin $\mathrm{D}$ concentration and chronic musculoskeletal system pain, which suggests a relationship between TTH and Vitamin D concentration. It is commonly known that Vitamin D levels decrease during winter months. On the contrary, the incidence of CTTH, depression, and musculoskeletal system symptoms increase during winter months, which is likely to be associated with decreased Vitamin D levels mainly because the pain TTH patients is known to be alleviated after Vitamin D supplementation (12-14). The aim of the present study was to examine the association between serum Vitamin D concentration and the severity of CTTH. 
Table 1. Descriptive statistics and comparison results for the three groups

\begin{tabular}{ccccc}
\hline & Group & $\mathrm{n}$ & Mean \pm Standard Deviation & $\mathrm{p}$ \\
\hline & $<10 \mathrm{ng} / \mathrm{mL}$ & 482 & $6.5102 \pm 2.01595 \mathrm{~b}$ & \\
Vitamin D3 & $10-25 \mathrm{ng} / \mathrm{mL}$ & 211 & $15.7831 \pm 3.89503 \mathrm{~b}$ & 0.001 \\
& $25>\mathrm{ng} / \mathrm{mL}$ & 89 & $44.8541 \pm 21.83026^{\mathrm{a}}$ & \\
\hline & Total & 782 & $12.0339 \pm 11.95700$ & \\
\hline
\end{tabular}

a, $b \downarrow$ : Different lower cases represent statistically significant differences among the groups

\section{Materials and Methods}

After obtaining an approval from the local ethics committee, patients aged 18 years or older that presented to our Neurology polyclinic with TTH during the winter of 2017-2018 were retrospectively reviewed. The patients were assigned into three groups according to Vitamin D concentration: (I) deficiency state: $<10 \mathrm{ng} / \mathrm{mL}$, (II) insufficiency state: 10-25 ng/mL, and (III) optimal state: $25-70 \mathrm{ng} / \mathrm{mL}$. The groups were compared with regard to age, gender, and Vitamin D levels. Tenderness in pericranial muscles (frontalis muscle, anterior temporalis muscle, and suboccipital muscles) was assessed by manual palpation and was graded between 0 and $3 \quad(0=$ no noticeable facial reaction or tenderness; $1=$ no noticeable facial reaction, but verbal expression of discomfort or mild pain; $2=$ verbal expression of painful tenderness with facial expression of discomfort; and $3=$ noticeable grimacing or withdrawal, verbal expression of pronounced painful tenderness). Serum D vitamin concentration was measured on an Abbott Architect I4000 SR immunoassay analyzer using the chemiluminescence microparticle immunoassay with an appropriate calibrator and kit. The results of serum Vitamin D measurements were expressed as $\mathrm{ng} / \mathrm{mL}$. The study included adult patients that presented to our polyclinic and were diagnosed with CTTH and excluded patients aged less than 18 years with acuteonset TTH, migraine, epilepsy, brain tumor Alzheimer's disease, Parkinson's disease, and stroke.

Statistical Analysis: Statistical evaluation of data was performed using SPSS version 16.0 (SPSS Inc. Co., Chicago, IL, USA). Descriptive statistics were expressed as mean \pm standard deviation (SD). For continuous variables, the comparison of group means was performed using One-Way ANOVA test followed by Duncan's multiple range test. Statistically significant level ( $\mathrm{p}$ value) was considered as $\% 5$.

\section{Results}

A total of 1,044 patients presented to our Neurology polyclinic during the winter of 2017-2018. Of these, 782 patients met the inclusion criteria and were included in the study. The patients comprised 32\% men and $68 \%$ women with a mean age of 34 years. Based on their serum Vitamin D levels, $482(61.6 \%)$ patients were classified into Group I $(<10 \mathrm{ng} / \mathrm{mL})$, $211(27.0 \%)$ patients into Group II $(10-25 \mathrm{ng} / \mathrm{mL})$, and $89(11.4 \%)$ patients into Group III $(>25 \mathrm{ng} / \mathrm{mL})$ and a significant difference was found among the three groups with regard to serum Vitamin D levels $(\mathrm{p}<0.01)$ (Table 1).

\section{Discussion}

The role of Vitamin D deficiency in headache was first reported in 1994 by Thys-Jacobs who presented two female cases of migraine (15). In later years, another study presented 8 cases of CTTH and reported that Vitamin D supplementation had contributory effects to CTTH (16). Headache is commonly accompanied by diffuse muscle pain. The incidence of chronic headache in patients with chronic musculoskeletal system symptoms is four times higher than that of individuals with no chronic musculoskeletal system symptoms. In patients with chronic musculoskeletal system symptoms, the headache can become a constituent of general body pain. On the other hand, accumulating evidence suggests that the comorbid conditions associated with pain semiology, muscular tenderness, other features, and CTTH are highly similar to the diffuse muscle pain caused by Vitamin D deficiency. Therefore, the coexistence of CTTH and general body pain in patients with Vitamin D deficiency is believed to have a mutual pathogenesis $(11,13,17)$. In a previous crosssectional study, Kjaergaard et al. showed significantly decreased serum 25-hydroxyvitamin D $(25[\mathrm{OH}] \mathrm{D})$ levels in patients with non-migraine headache (17). In our study, $88 \%$ of the patients had a decreased serum Vitamin D level (Table 1) and a significant difference was found among the three groups $(p<0.001)$. In another cross-sectional study, Knusten et al. found decreased serum $25(\mathrm{OH})$ D3 levels in patients with musculoskeletal system pain, fatigue, and headache (18).

Limitations: The present study was limited as it was designed as a retrospective single-center study that reviewed the patients who presented to an adult 
Neurology clinic in a tertiary health institution. Therefore, the results of the study may not be generalized to the general population and the study may fail to represent all CTTH patients due to the mode of hospital admission and biases. Another limitation was that no pain assessment was performed via Visual Analog Scale (VAS).

Vitamin D deficiency is an increasingly major health problem due to modern lifestyle. Accordingly, Vitamin D deficiency should be considered in patients presenting with CTTH. Serum Vitamin D concentration may change depending on various factors. Therefore, further multicenter studies are needed to investigate whether serum Vitamin D levels vary across settings.

\section{References}

1. Trkanjec Z, Aleksić-Shihabi A.Acta Med Croatica. Tension-type headaches2008; 62: 205-210.

2. Solomon GD. Chronic tension-type headache: advice for the viselike-headache patient. Cleve Clin J Med 2002; 69: 167-172.

3. Fumal A, Schoenen J. Tension-type headache: Current research and clinical management. Lancet Neurol 2008; 7: 70-83.

4. Headache Classification Committee of the International Headache S. The International Classification of Headache Disorders, 3rd edition (beta version). Cephalalgia 2013; 33: 629808.

5. Jensen R. Peripheral and central mechanisms in tension-type headache: an update. Cephalalgia 2003; 23: 49-52.

6. Vitamin D deficiency. Holick MF. N Engl J Med 2007; 357: 266-281.

7. Holick MF, Chen TC. Vitamin D deficiency: a worldwide problem with health consequences. Am J Clin Nutr 2008; 87: 1080-1086.

8. Straube S, Andrew Moore R, Derry S, McQuay HJ. Vitamin D and chronic pain. Pain 2009; 141: 10-13.
9. Wheeler SD. Vitamin D deficiency in chronic migraine. Headache 2008; 48: 52-53.

10. Schoenen J, Bottin D, Hardy F, Gerard P. Cephalic and extracephalic pressure pain thresholds in chronic tension-type headache. Pain 1991; 47: 145-149.

11. Hu JW, Sessle BJ, Raboisson P, Dallel R, Woda A. Stimulation of craniofacial muscle afferents induces prolonged facilitatory effects in trigeminal nociceptive brain-stem neurones. Pain 1992; 48: 53-60.

12. Prakash S, Shah ND. Chronic tension-type headache with vitamin $\mathrm{D}$ deficiency: Casual or causal association? Headache 2009; 49: 12141222.

13. Prakash S, Kumar M, Belani $\mathrm{P}$ et al. Interrelationships between chronic tensiontype headache, musculoskeletal pain, and vitamin $\mathrm{D}$ deficiency: is osteomalacia responsible for both headache and musculoskeletal pain? Ann Indian Acad Neurol 2013; 16: 650-658.

14. Prakash S, Rathore C, Makwana P, Dave A, Joshi H, Parekh H. Vitamin D Deficiency in Patients With Chronic Tension-Type Headache: A CaseControl Study. Headache 2017; 57: 1096-1108.

15. Thys-Jacobs S. Alleviation of migraines with therapeutic vitamin D and calcium. Headache 1994; 34: 590-592.

16. Prakash S, Makwana P, Rathore C. Vitamin D deficiency mimicking chronic tension-type headache in children. BMJ Case Rep 2016; 2: 2016.

17. Kjaergaard M, Eggen AE, Mathiesen EB, Jorde R. Association Between Headache and Serum 25Hydroxyvitamin D; the Tromsø Study: Tromsø 6. Headache 2012; 10: 1499-1505.

18. Knutsen KV, Madar AA, Brekke M, et al. Effect of vitamin $\mathrm{D}$ on musculoskeletal pain and headache: A randomized, double-blind, placebocontrolled trial among adult ethnic minorities in Norway Pain 2014; 155: 2591-2598. 